\title{
USO DO CILINDRO INFILTRÔMETRO ÚNICO EM DIFERENTES SOLOS
}

\section{WELSON L. SIMÕES ${ }^{1}$, VLADIMIR B. FIGUEIRÊDO ${ }^{2}$, ELIO L. DA SILVA ${ }^{3}$}

RESUMO: O infiltrômetro de anéis concêntricos (IAC) tem sido empregado para a determinação da velocidade de infiltração básica de água no solo (VIB), porém, para sistemas de irrigação por aspersão, a melhor técnica é a que apresenta carga hidráulica zero, como nos simuladores de chuva. O presente trabalho teve o objetivo de avaliar o uso de infiltrômetro de cilindro único (ICU), comparando-o com o IAC e com um simulador de chuva. O experimento foi realizado em dois tipos de solo classificados como Latossolo Vermelho distroférrico e Cambissolo, onde se utilizou um ICU com 0,20 m de diâmetro e $0,50 \mathrm{~m}$ de altura. As cargas hidráulicas utilizadas variaram de $0,03 \mathrm{~m}$ a $0,30 \mathrm{~m}$ com intervalos crescentes de $0,03 \mathrm{~m}$, com três repetições. Utilizou-se de um IAC de 0,30 e $0,90 \mathrm{~m}$ de diâmetro, com três repetições, nas cargas hidráulicas mais utilizadas no campo, 0,05 m e 0,10 m. O simulador de chuva utilizado teve área útil de $1,0 \mathrm{~m}^{2}$ com dois difusores. Com o aumento da carga hidráulica para o ICU, o fluxo de entrada de água no solo aumentou de forma polinomial para os dois solos. Para o IAC, a VIB pode ser subestimada ou superestimada, dependendo do solo e da carga hidráulica. Pode-se estimar a VIB, para uma carga hidráulica zero, utilizando-se de um ICU na carga desejada, desde que se aplique o fator de correção inerente de cada tipo de solo.

PALAVRAS-CHAVE: infiltrômetro, infiltração, irrigação.

\section{USE OF SINGLE RING INFILTROMETER IN DIFFERENT SOILS}

\begin{abstract}
Infiltrometer ring (IAC) has been the most used way for the soil intake rate determination, although the best result comes from the technique using no pressure head, like in rainfall simulator, for application in sprinkler irrigation. The objective of this work was the evaluation of a single ring infiltrometer (ICU) comparing its data with those from an IAC and rainfall simulator. The evaluation was done in two types of soils, Dystrophic Dusky Red Latosol and Cambisol, using an ICU with diameter of $0.20 \mathrm{~m}$ and $0.50 \mathrm{~m}$ high, with hydraulic heads varying from $0.03 \mathrm{~m}$ to $0.30 \mathrm{~m}$ with intervals of $0.03 \mathrm{~m}$ and three repetitions. It was used, for comparison, an IAC with diameters of $0.30 \mathrm{~m}$ and $0.90 \mathrm{~m}$, with three replications and hydraulic heads of $0.05 \mathrm{~m}$ and $0.10 \mathrm{~m}$. The rainfall simulator utilized had an area of $1.0 \mathrm{~m}^{2}$ with two sprinklers. It was observed that, the elevation of hydraulic head in the ICU promoted an increase in the water flow into the soil, in a polynomial model, for both soils. The IAC may underestimate or upperestimate the real values of final intake rate, depending on the soil and the hydraulic head. The soil water intake rate can be estimate, with no pressure head, using an ICU with a desired pressure since a correction factor is applied according to the soil type.
\end{abstract}

KEYWORDS: infiltrometer, intake rate, irrigation.

\footnotetext{
${ }^{1}$ Eng ${ }^{\circ}$ Agrônomo, Mestrando em Engenharia Agrícola/Irrigação e Drenagem, Universidade Federal de Lavras, UFLA, Departamento de Engenharia, Lavras - MG, Fone: (0XX35) 3822-5587, welsimoes@ yahoo.com.br

${ }^{2}$ Eng- Agrônomo, M.Sc. em Engenharia Agrícola/Irrigação e Drenagem, Departamento de Engenharia, UFLA, Lavras - MG.

${ }^{3}$ Eng ${ }^{0}$ Agrônomo, M.Sc., Ph.D., Departamento de Engenharia, UFLA, Lavras - MG.

Recebido pelo Conselho Editorial em: 24-11-2003

Aprovado pelo Conselho Editorial em: 21-1-2005
} 


\section{INTRODUÇÃO}

Uma das principais preocupações na avaliação da infiltração da água no solo é a escolha de um método adequado ao tipo de irrigação. A utilidade de cada método está relacionada à finalidade dos dados, à precisão desejada, à facilidade de manejo e ao custo operacional. Os métodos mais utilizados são: entrada e saída de água no sulco, infiltrômetro de anéis concêntricos e simuladores de chuva. $\mathrm{O}$ infiltrômetro de anéis concêntricos é o mais utilizado devido a sua facilidade de manejo e ao baixo custo. Porém, segundo BOWER (1986), esse método tem suas limitações quando se deseja levantar dados de infiltração para fins de elaboração de projetos de irrigação por aspersão. Esse infiltrômetro apresenta particularidades que podem resultar em erros na determinação da infiltração, uma vez que sua operação se dá na presença de uma carga hidráulica acima do solo, simulando as condições de inundação, não representando, portanto, as condições de irrigação por aspersão.

Para fins de irrigação, tem-se considerado a velocidade de infiltração básica de água no solo como sendo o fluxo constante ("steady-state flow") de entrada de água em uma área delimitada por dois cilindros concêntricos. Têm-se utilizado valores de carga hidráulica (altura de água sobre a superfície do solo) sem muito critério, com a única preocupação de que devem ser os menores possíveis. Diferentes valores de carga hidráulica promovem diferentes valores de fluxo constante que, erroneamente, têm sido considerados como infiltração básica. Para atender à definição de infiltração, a carga hidráulica do infiltrômetro deveria ser zero (pressão atmosférica), o que tornaria impraticável a utilização de infiltrômetro de anéis concêntricos (IAC), ou levaria à necessidade de correção do valor constante de fluxo para ter a infiltração básica. A carga hidráulica zero pode ser conseguida, na prática, com o uso de um simulador de chuva; no entanto, esse aparelho apresenta alto custo de aquisição e operacionalidade mais complexa, uma vez que necessita de pressão de serviço para funcionamento dos aspersores e nem sempre se dispõe dessa condição em campo.

Outros erros podem existir na determinação da infiltração de água no solo, devido, também, ao fluxo lateral de água no solo abaixo do infiltrômetro. Segundo HAISE et al. (1956), citado por SKAGGS et al. (1983), uma forma de evitar esses erros é utilizar IAC, tendo o cilindro externo a função de evitar o fluxo lateral da água no solo. Para SKAGGS et al. (1983), a relação entre os diâmetros dos cilindros, externo e interno, deve ser igual ou superior a 2. BOUWER (1986), no entanto, discorda da necessidade de um cilindro externo, afirmando que a única forma de medir a infiltração vertical de um solo é utilizando cilindros com diâmetros suficientemente grandes de maneira que a relação entre a pressão de entrada de ar do solo e o diâmetro do cilindro seja praticamente nula. Nessa condição, a infiltração em todos os pontos dentro do cilindro será bem próxima da infiltração vertical. Em estudos realizados por SWARTZENDRUBER \& OLSON (1961), com solo arenoso, essa situação foi alcançada quando se utilizou de um cilindro com 1,20 m de diâmetro. Considerando-se que a areia apresenta os menores valores de pressão de entrada de ar, conclui-se que, para solos não-arenosos, os cilindros devam ter diâmetros superiores a 1,20 m, o que inviabiliza o uso do método para a determinação da infiltrabilidade de solos.

Segundo MERRIAN et al. (1983), pode-se utilizar um infiltrômetro de cilindro único (ICU) na determinação da infiltração vertical desde que ele tenha, no mínimo, 0,25 $\mathrm{m}$ de diâmetro e que seja instalado a, pelo menos, $0,15 \mathrm{~m}$ abaixo da superfície do solo. Cilindros concêntricos podem ser utilizados, porém raramente se consegue melhor precisão.

Pretendeu-se, com o presente estudo, determinar a correção necessária para ter a infiltração básica de água no solo, com "carga zero", a partir dos fluxos constantes obtidos com cargas hidráulicas crescentes em infiltrômetro de cilindro único de $0,20 \mathrm{~m}$ de diâmetro e com o simulador de chuva. Dessa forma, pode-se utilizar a carga hidráulica que julgar mais conveniente, sabendo-se da necessidade e possibilidade de correção do resultado, para obter o valor real da infiltração básica. 


\section{MATERIAL E MÉTODOS}

O experimento foi realizado em dois solos classificados como Latossolo Vermelho distroférrico e Cambissolo, sem camada física de impedimento, cujas características texturais estão apresentadas na Tabela 1.

TABELA 1. Características texturais dos solos Latossolo Vermelho distroférrico e Cambissolo, nas camadas de 0-0,20 m e 0,20-0,40 m.

\begin{tabular}{lcccc}
\hline & \multicolumn{2}{c}{ Latossolo Vermelho distroférrico } & \multicolumn{2}{c}{ Cambissolo } \\
\hline & $0-0,20 \mathrm{~m}$ & $0,20-0,40 \mathrm{~m}$ & $0-0,20 \mathrm{~m}$ & $0,20-0,40 \mathrm{~m}$ \\
\cline { 2 - 5 } Areia & $13 \%$ & $13 \%$ & $7 \%$ & $6 \%$ \\
Silte & $30 \%$ & $28 \%$ & $32 \%$ & $37 \%$ \\
Argila & $57 \%$ & $59 \%$ & $61 \%$ & $57 \%$ \\
\hline
\end{tabular}

Testou-se um infiltrômetro de cilindro único (ICU) com 0,20 m de diâmetro e 0,50 m de altura, feito com chapa galvanizada, sendo a parte inferior em forma de bisel para facilitar a instalação e evitar grandes alterações na estrutura do solo. Para avaliação do fluxo constante de entrada de água pela superfície do solo sob diferentes cargas hidráulicas, utilizou-se de um controlador de carga do tipo Frasco de Mariotte, conectado ao ICU. As cargas hidráulicas utilizadas nesses testes variaram de $0,03 \mathrm{~m}$ a $0,30 \mathrm{~m}$, com intervalos crescentes de $0,03 \mathrm{~m}$, com três repetições. Para fins de comparação, utilizou-se do método tradicional do infiltrômetro de anéis concêntricos (IAC), segundo BERNARDO (1995), com 0,40 m de altura, tendo o maior diâmetro $0,90 \mathrm{~m}$ e o menor diâmetro $0,30 \mathrm{~m}$, utilizando-se também de três repetições, com as cargas hidráulicas mais utilizadas no campo, 0,05 e 0,10 m, também controladas pelo regulador de carga do tipo Frasco de Mariotte. Todos os cilindros foram instalados no solo até a profundidade de 0,15 m, conforme recomendações de MERRIAN et al. (1983). Realizaramse os testes até que o fluxo de entrada de água no solo se tornasse constante em cada carga hidráulica testada.

Testou-se, também, um simulador de chuva (Figura 1), com área útil de 1,0 $\mathrm{m}^{2}$, devidamente limitada por chapas de aço $\mathrm{n}^{\mathrm{o}} 18$, de $0,25 \mathrm{~m}$ de altura, dos quais $0,15 \mathrm{~m}$ foram cravados no solo. $\mathrm{O}$ aparelho tinha altura de $0,80 \mathrm{~m}$, com dois difusores de intensidade de precipitação igual a $148 \mathrm{~mm} \mathrm{~h}^{-1}$,

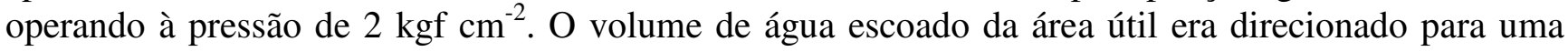
calha transversal e abaixo da superfície do solo que, acoplado a uma mangueira, transportava a água para uma parte mais baixa do terreno, longe da precipitação dos difusores, onde era feita a quantificação em determinados intervalos de tempo. A VIB foi obtida quando o volume escoado se tornou constante com o tempo.

Para a obtenção dos fatores de correção, dividiu-se o valor da VIB encontrado para o simulador de chuva pelo valor encontrado para cada fluxo constante obtido e ajustou-se uma regressão com base no melhor coeficiente de determinação $\left(\mathrm{r}^{2}\right)$, para cada solo estudado. 


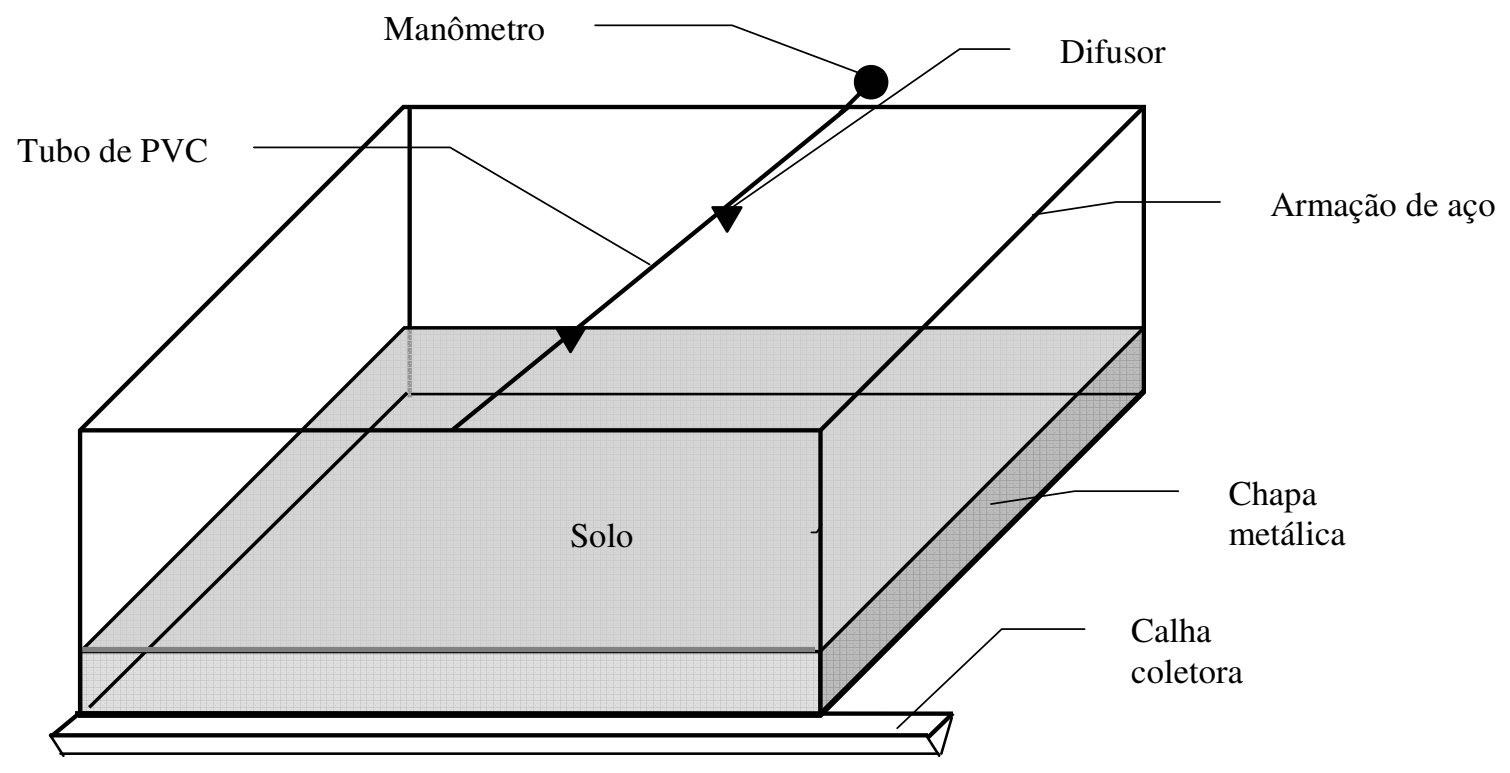

FIGURA 1. Visão geral do simulador de chuva com seus componentes instalados.

\section{RESULTADOS E DISCUSSÃO}

Na Figura 2, mostram-se os resultados obtidos para o Latossolo, em que a VIB encontrada pelo método do simulador de chuva foi de $42,0 \mathrm{~mm} \mathrm{~h}^{-1}$ e os fluxos constantes para IAC com cargas de

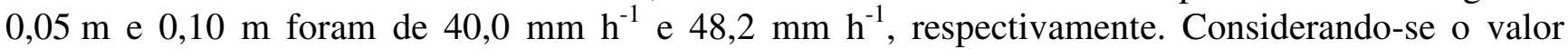
obtido pelo método do simulador de chuva como ideal, observa-se que, para a carga hidráulica de 0,10 m, o valor da VIB é superestimado em 14,8\% e, para carga hidráulica de 0,05 m, obteve-se valor de fluxo constante 5\% menor que a VIB. Observa-se, também, nessa figura, que, para o ICU, o acréscimo da carga hidráulica aumentou de forma polinomial ao fluxo de entrada de água no solo, e todas as cargas hidráulicas testadas proporcionaram fluxo constante superior à VIB encontrada no simulador (Figura 2).

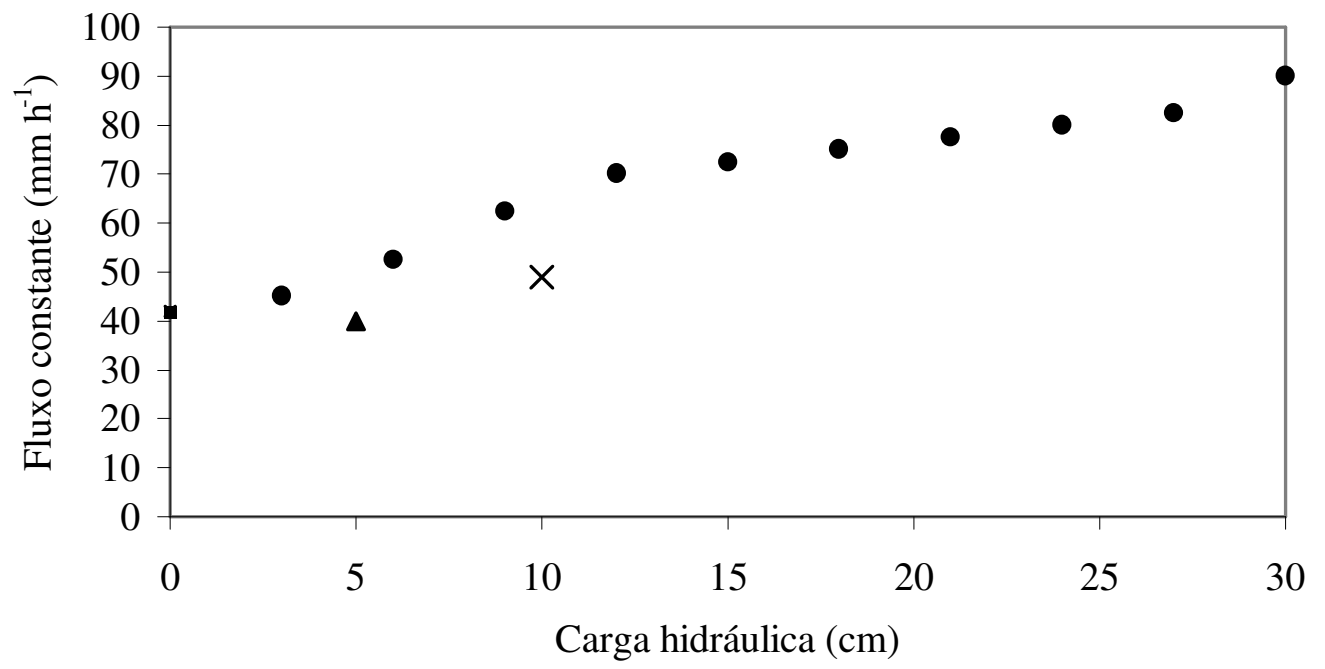

- ICU $\triangle \mathrm{IAC}(5 \mathrm{~cm}) \quad \times \operatorname{IAC}(10 \mathrm{~cm}) \quad$ - Simulador de chuva

FIGURA 2. Fluxo constante de água no solo, em $\mathrm{mm} \mathrm{h}^{-1}$, do ICU, IAC e simulador de chuva, com as cargas hidráulicas testadas em cada método, para o Latossolo Vermelho distroférrico. 
Os altos valores de VIB observados para esse solo têm relação com seu material originário que, segundo ROCHA (1982), é rocha eruptiva básica, tendo como característica marcante alta capacidade de infiltração, devido à sua estrutura, apesar de ser um solo com alto teor de argila.

A subestimação não-esperada da VIB encontrada para a carga $0,05 \mathrm{~m}$ do IAC, com relação ao simulador de chuva, não foi significante a $5 \%$, pelo teste de Tukey. Esse fato pode estar associado à variabilidade espacial natural do solo. Os demais resultados corroboram os encontrados por COSTA et al. (1999), que, para essa mesma classe de solo, comparou a VIB obtida por um IAC (usando carga hidráulica de $0,08 \mathrm{~m}$ ) com a obtida por um simulador de chuva, em que o último apresentou VIB ligeiramente menor. COELHO et al. (2000) também encontraram superestimação da VIB pelo IAC com relação ao simulador de chuva para Terra Roxa Estruturada.

Por meio do ajuste de um modelo polinomial aos dados do ICU [eq.(1)], com $\mathrm{r}^{2}=0,969$, simularam-se os valores das cargas utilizadas no IAC $(0,05 \mathrm{~m}$ e $0,10 \mathrm{~m})$, encontrando-se os valores $51,53 \mathrm{~mm} \mathrm{~h}^{-1}$ e $62,47 \mathrm{~mm} \mathrm{~h}^{-1}$, respectivamente, os quais são $28,8 \%$ e $29,6 \%$ superiores, respectivamente. Essas diferenças podem estar associadas à grande variabilidade espacial para esse atributo, observada por COELHO et al. (2000), que reafirmam a necessidade de um ajuste para a obtenção da VIB em ambas as metodologias.

$$
y=-0,04 x^{2}+2,7891 x+38,583
$$

em que,

$\mathrm{y}$ - fluxo constante, $\mathrm{mm} \mathrm{h}^{-1}$, e

$\mathrm{x}$ - carga hidráulica utilizada, $\mathrm{cm}$.

Na Figura 3, apresentam-se os dados obtidos para o Cambissolo, em que a VIB encontrada pelo método do simulador de chuva foi $57,68 \mathrm{~mm} \mathrm{~h}^{-1}$ e os fluxos constantes para o IAC com cargas de 0,05 $\mathrm{m}$ e $0,10 \mathrm{~m}$ foram $104,44 \mathrm{~mm} \mathrm{~h}^{-1}$ e $143,33 \mathrm{~mm} \mathrm{~h}^{-1}$, respectivamente. Considerando-se o valor obtido pelo método do simulador de chuva como ideal, observa-se que o valor da VIB foi superestimado em $81,07 \%$ e 148,49\%, respectivamente. Observa-se, também, nessa Figura, que, para o cilindro-teste, o incremento da carga hidráulica aumentou de forma polinomial ao fluxo de entrada de água no solo e todas as cargas hidráulicas testadas proporcionaram fluxos constantes superiores à VIB encontrada no simulador.

Os altos valores de VIB observados para o Cambissolo corroboram os resultados obtidos por OTTONI FILHO (2003) que, para quatro Cambissolos estudados, encontrou valores de VIB superior a $260 \mathrm{~mm} \mathrm{~h}^{-1}$, resultado cinco vezes maior que o observado pelo mesmo autor para um solo arenoso. É possível que a alta permeabilidade observada nesse solo, aliada ao uso de cargas hidráulicas, como usado no IAC, tenha contribuído para as grandes superestimações no valor da VIB.

Por meio do ajuste de um modelo polinomial aos dados do ICU [eq.(2)], com $\mathrm{r}^{2}=0,9902$, simularam-se os valores das cargas utilizadas no IAC $(0,05 \mathrm{~m}$ e $0,10 \mathrm{~m})$, encontrando-se valores de fluxo de $80,61 \mathrm{~mm} \mathrm{~h}^{-1}$ e $99,3 \mathrm{~mm} \mathrm{~h}^{-1}$, respectivamente, os quais subestimam os valores encontrados no IAC, em $29,56 \%$ e $44,34 \%$, respectivamente.

$$
y=0,1915 x^{2}+0,8649 x+71,5
$$

em que,

$$
\begin{aligned}
& \text { y - fluxo constante, } \mathrm{mm} \mathrm{h}^{-1}, \mathrm{e} \\
& \mathrm{x} \text { - carga hidráulica utilizada, } \mathrm{cm} \text {. }
\end{aligned}
$$




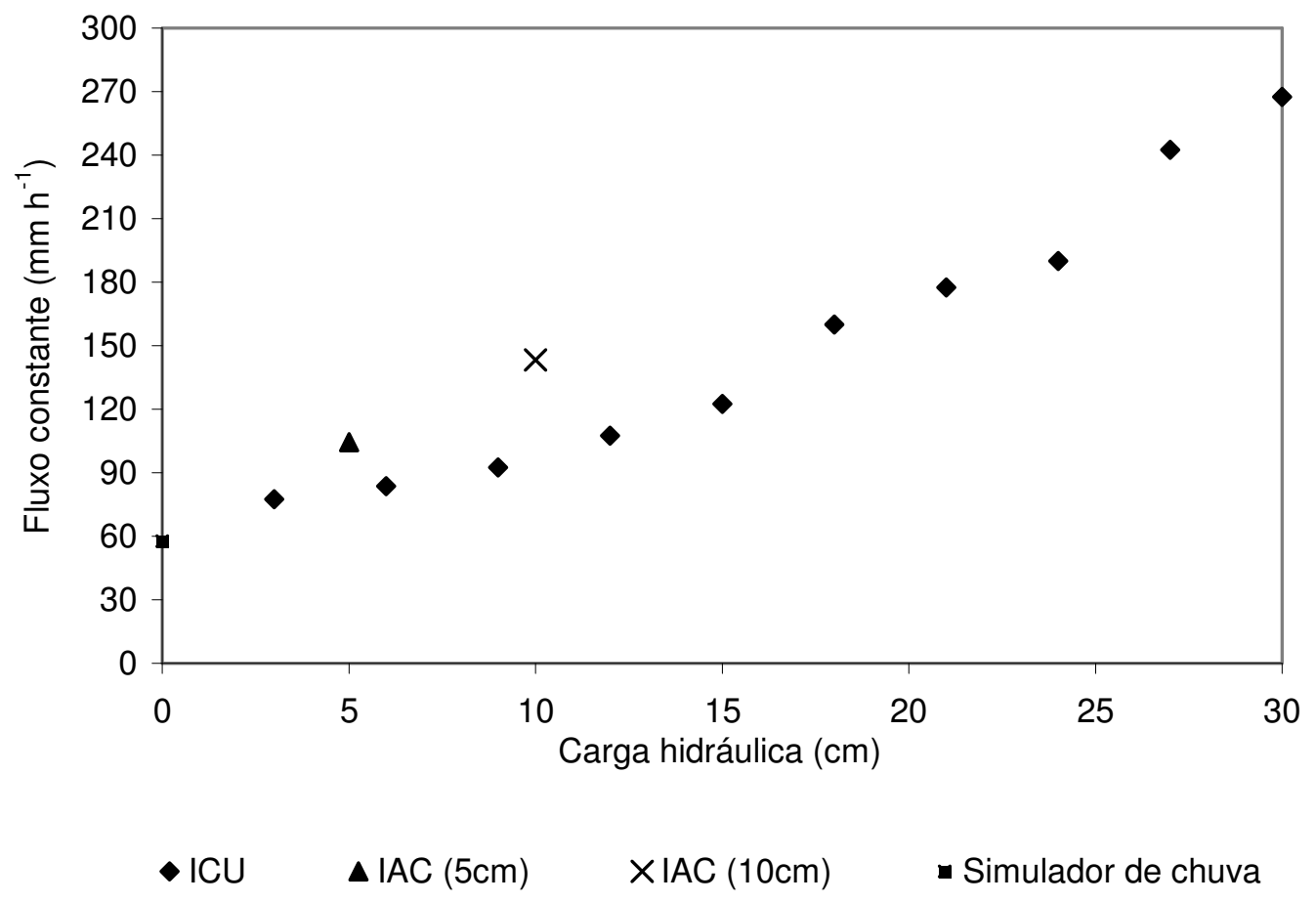

FIGURA 3. Fluxo constante em $\mathrm{mm} \mathrm{h}^{-1}$, do ICU, IAC e simulador de chuva, com as cargas hidráulicas testadas em cada método, para o Cambissolo.

Observou-se que o comportamento do fluxo constante obtido pelo IAC, com relação ao simulador, foi variável em função do tipo de solo. Para o ICU, obtiveram-se valores de fluxo constante sempre acima dos valores encontrados no simulador, resultado coerente, uma vez que se utilizou de carga hidráulica maior. Com os modelos gerados para os dados do ICU, para os dois solos, simulou-se a carga hidráulica zero e obteve-se diferença de apenas 8,8\% para o Latossolo e 23,9\% para o Cambissolo, quando comparadas com os valores encontrados pelo simulador de chuva, o que indica certa coerência dos valores encontrados e reforça a viabilidade de uso de cilindro único.

Na Figura 4, mostra-se o comportamento dos fatores de correção para os fluxos com as diferentes cargas hidráulicas utilizadas no ICU, em relação à VIB encontrada no simulador de chuva para os dois solos em estudo, em que os modelos de melhor ajuste foram do tipo potencial [eq.(3)], com $\mathrm{r}^{2}=0,984$ para o Latossolo, e para o Cambissolo [eq.(4)], com $\mathrm{r}^{2}=0,9879$. Com o uso dessas equações, para cada tipo de solo, transforma-se o fluxo encontrado com a carga utilizada nos testes, em valor de VIB que seria obtido se o teste fosse realizado com simulador de chuva ("situação ideal" para sistemas de irrigação que não utilizem carga hidráulica).

$$
\begin{aligned}
& y=1,2912 x^{-0,2904} \\
& y=0,9198 e^{-0,0484 x}
\end{aligned}
$$

em que,

y - fator de correção, e

x - carga hidráulica utilizada, $\mathrm{cm}$. 


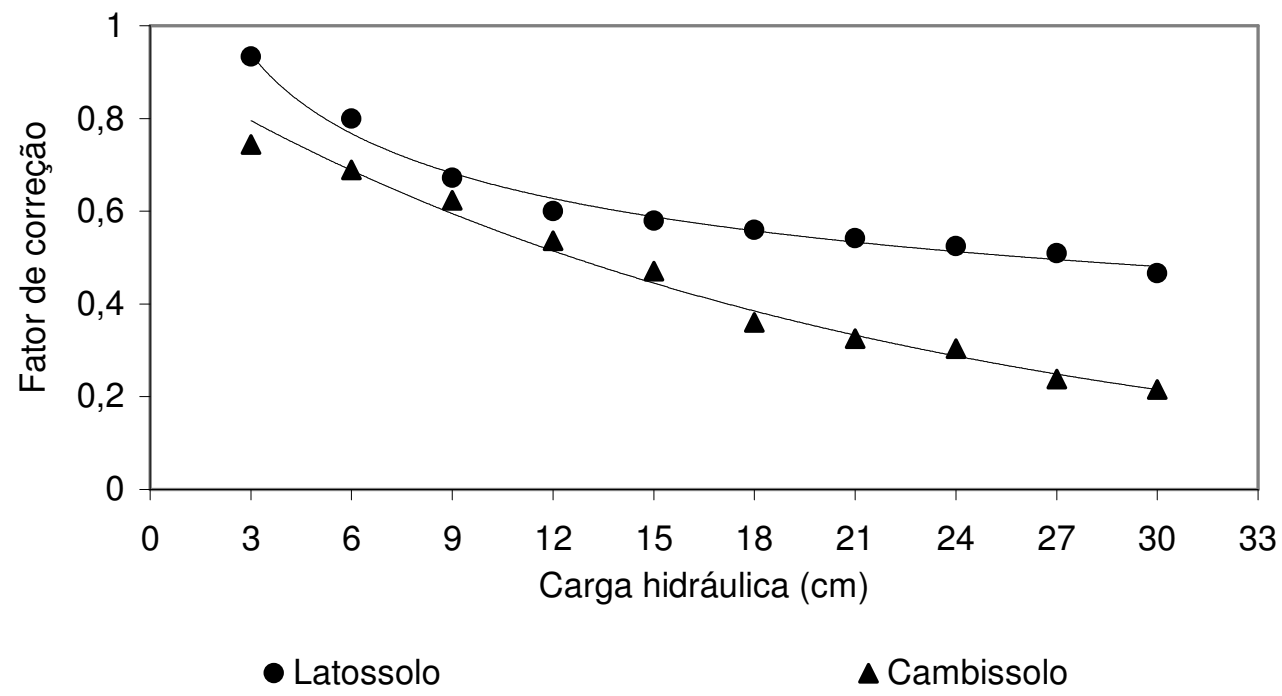

FIGURA 4. Fatores de correção para a VIB obtidos com as cargas hidráulicas utilizadas nos testes para os solos Latossolo Vermelho distroférrico e Cambissolo, com as respectivas linhas de ajuste.

\section{CONCLUSÕES}

A VIB obtida pelo uso do IAC pode superestimar ou subestimar o valor da VIB obtida por simulador de chuva, dependendo do solo e da carga hidráulica utilizada.

É possível estimar a VIB, utilizando-se de ICU com a carga hidráulica desejada, desde que, para isso, se aplique um fator de correção, o qual depende do tipo de solo em estudo.

\section{REFERÊNCIAS}

BERNARDO, S. Manual de irrigação. Viçosa: UFV, Imprensa Universitária, 1995. 596 p.

BOUWER, H. Intake Rate: Cylinder Infiltrometer. In: KLUTE (Ed.). Methods of soil analysis, Part 1 Physical and mineralogical methods. Madison: ASA, SSSA, 1986. p.825-44. (Agronomy Monography, 9, Book Series: 5))

COELHO, R.D.; MIRANDA, J.H. de; DUARTE, S.N. Infiltrômetro de anéis versus infiltrômetro de aspersores. Revista Brasileira de Engenharia Agrícola e Ambiental, Campina Grande, v.4, n.2, p.137$41,2000$.

COSTA, E.L.; SILVA, A.M. da; COLOMBO, A.; ABREU, A.R. de. Infiltração de água em solo, determinada por simulador de chuvas e pelo método dos anéis. Revista Brasileira de Engenharia Agrícola e Ambiental, Campina Grande, v.3, n.2, p.131-4, 1999.

MERRIAN, J.L.; SHEARER, M.N.; BURT, C.M. Evaluating irrigation systems and practices. In: JENSEN, M.E. (ed.) Design and operations of farm irrigation systems. St Joseph: ASAE, 1983. p.72160. (Monography Series, 3)

OTTONI FILHO, T.B. Uma classificação físico-hídrica dos solos. Revista Brasileira de Ciência do Solo, Campinas, v.27, n.2, p.211-22, 2003.

ROCHA, G.C. Geologia, geomorfologia e pedologia de uma catena de solos, situada no Câmpus da Escola Superior de Lavras- MG. 1982. 109 f. Dissertação (Mestrado em Solos e Nutrição de Plantas) Escola Superior de Agricultura de Lavras, Lavras, 1982. 
SKAGGS, R.W.; KILLER, D.E.; BROOKS, R.H. Soil Water. In: JENSEN, M.E. (ed.) Design and operations of farm irrigation systems. St Joseph: ASAE, 1983. p.77-123. (Monography Series, 3) SWARTZENDRUBER, D.; OLSON, T.C. Model study of the double ring infiltrometer as affected by depth of wetting and particle size. Soil Science, Baltimore, v.92, n.1, p.219-25, 1961. 